\title{
Size-exclusion chromatography can identify faster-associating protein complexes and evaluate design strategies Chad L Mayer ${ }^{1,2}$, W Kalani Snyder ${ }^{1}$, Monika A Swietlicka ${ }^{1}$, Andrew D VanSchoiack ${ }^{1}$, Chad R Austin ${ }^{1,3}$ and Benjamin J McFarland*1
}

Address: ${ }^{1}$ Department of Chemistry and Biochemistry, Seattle Pacific University, 3307 Third Avenue West, Seattle, WA 98119-1997, USA, ${ }^{2}$ Current address : Seattle Biomedical Research Institute, Seattle, WA 98109, USA and ${ }^{3}$ Current address : Dept. of Microbiology, University of Colorado Denver, Aurora, CO 80045, USA

Email: Chad L Mayer - mayerc@spu.edu; W Kalani Snyder - kalani@spu.edu; Monika A Swietlicka - swietm@spu.edu; Andrew D VanSchoiack - vansca@gmail.com; Chad R Austin - chad.austin@ucdenver.edu; Benjamin J McFarland* - bjm@spu.edu

* Corresponding author

Published: 15 July 2009

BMC Research Notes 2009, 2:135 doi:10.1186/1756-0500-2-135
Received: 15 June 2009

Accepted: I5 July 2009

This article is available from: http://www.biomedcentral.com/1756-0500/2//35

(c) 2009 McFarland et al; licensee BioMed Central Ltd.

This is an Open Access article distributed under the terms of the Creative Commons Attribution License (http://creativecommons.org/licenses/by/2.0), which permits unrestricted use, distribution, and reproduction in any medium, provided the original work is properly cited.

\begin{abstract}
Background: We previously developed a set of rationally designed mutant MICA protein ligands for the NKG2D immunoreceptor in which MICA was mutated at residues that do not contact NKG2D. Some of these MICA mutants, predicted by RosettaDesign to be destabilized, bound NKG2D with affinities enhanced by more than an order of magnitude when evaluated by surface plasmon resonance (SPR).

Findings: Small-zone size-exclusion chromatography (SEC) detected persistent high-affinity MICA mutant-NKG2D complexes in solution as early-eluting peaks. The SEC binding assay used standard protein purification instrumentation to evaluate complex stability, qualitatively paralleled the SPR results, and successfully discriminated among complexes that differed only in on-rates. We used the SEC binding assay, along with SPR, to assess the results of a follow-up design strategy targeting the non-interfacial redesigned region. Both SEC and SPR agreed that these mutations did not enhance affinity as much as previous mutants. When the SEC binding assay was run in I $M$ urea, only the highest affinity complex was detected.

Conclusion: This SEC binding assay provides a correlation with SPR results for protein complex affinities, detecting changes in complex on-rates, and tunable to lower sensitivity with I M urea. The SEC binding assay is complementary to other protein design evaluation methods, can be adapted to the undergraduate research laboratory, and may provide additional structural information about changes in hydrodynamic radii from elution times. Our assay allowed us to conclude that further alteration of MICA at non-contacting residues is unlikely to further enhance NKG2D affinity.
\end{abstract}

\section{Background}

Several protein design algorithms have been produced over the past decade for rationally altering and optimizing the cores of proteins, protein-ligand interfaces, and protein-protein interfaces for structural and therapeutic application [1], including RosettaDesign [2]. Protein 
design schemes require a step for screening a set of candidate proteins. Size-exclusion chromatography (SEC) is an option for design evaluation if a protein's size is changed significantly or a larger, high-affinity protein-protein complex is formed. For protein-protein complexes, the SEC column can be saturated with protein for a large-zone assay [3], or a small plug of protein can be injected onto a column for a small-zone assay [4,5]. Small-zone techniques have been used for the MICA-NKG2D protein-protein interaction we investigate in this paper using small (analytical) amounts of protein and detecting the presence or absence of persistent complexes [6], as a purification step for MICA-NKG2D complex crystallization [7], and for diverse ends with other proteins [8-10].

SEC has the technical advantages of preparative scale, speed and cost-effectiveness relative to binding techniques such as surface plasmon resonance (SPR). In addition, persistence of a complex through an SEC column implies that the proteins physically adhered or equilibrated quickly enough to effectively adhere on a scale of minutes to hours, even as unbound molecules were partitioned away by the action of the column. This physical evidence that the protein-protein complex has been maintained over a long time shows that the complex has a significant "residence time," a concept which has proved useful for small-molecule drugs [11] and may be useful for finding or characterizing potent designed-protein drugs or receptor-ligand complexes [12].

Here we describe the full results a small-zone analytical SEC protein-protein binding assay developed to probe the design space for the MICA-NKG2D interaction, which was mentioned but not detailed in a previous publication [13]. MICA binds homodimeric NKG2D on the surface of certain immunocytes, triggering the death of stressed cells such as transformed cancerous cells [14]. We previously described how a set of 25 mutant MICA proteins was made in which eight MICA residues were varied [13]. These mutations were not located directly at the NKG2DMICA binding interface, but were below it, located within and under the $\alpha 2$ helix (Figure 1). This atypical design strategy produced unexpected results: MICA proteins with lower design scores, predicting more stable MICA proteins, bound NKG2D with the same or weaker affinity, while some MICA proteins with higher design scores, predicting that the MICA proteins themselves would be less stable, bound NKG2D with greater affinity [13]. Here we report the complete results of our SEC binding assay and correlate them with the previously reported thermodynamic and kinetic results [13] obtained by surface plasmon resonance (SPR). We also extend the data set with follow-up MICA designs, bringing to conclusion our analysis of destabilizing design strategies.
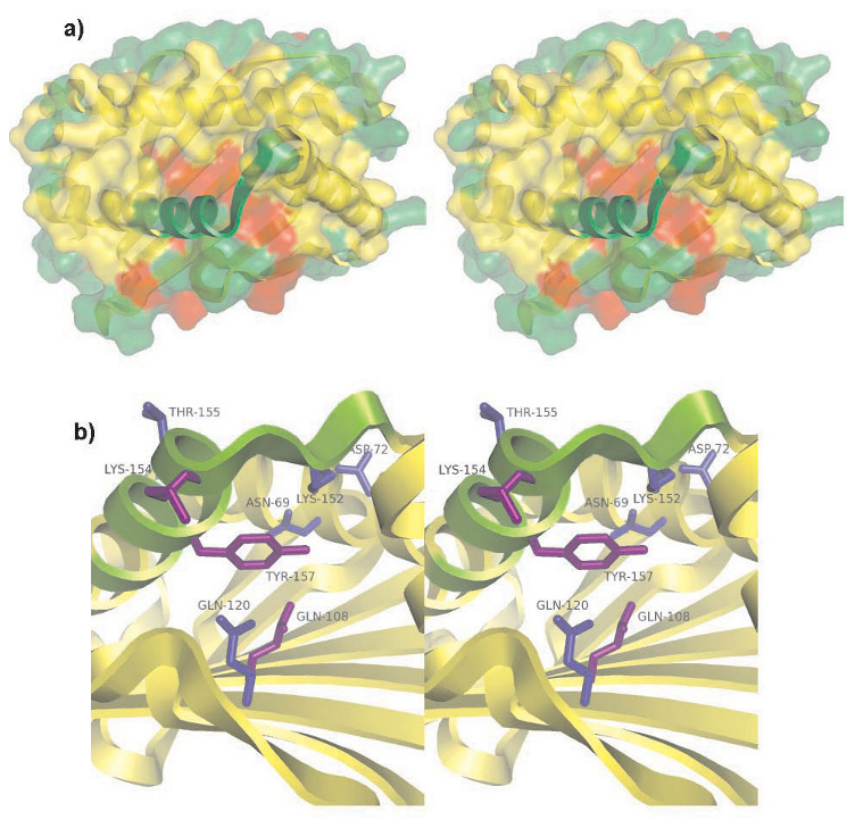

Figure I

Location of disordered region and design mutations in the MICA structure. (a) The receptor-bound structure of MICA (Protein Data Bank ID IHYR chain C) in stereo, shown as ribbons and colored by PDB-assigned secondary structure of unbound MICA (PDB ID IB3]), with beta sheets red, helices yellow and loops green. (Residues in the $\alpha 2$ helix of receptor-bound MICA that are absent from the crystal structure of unbound MICA are colored green.) The solventaccessible surface of unbound MICA is overlaid on the receptor-bound structure with the same coloring, showing how the disordered region exposes the underlying beta-sheet. (b) Side view in stereo of the receptor-bound wild-type MICA structure used by RosettaDesign, with the helix backbone that is not observed in the unbound structure colored green. The eight redesigned residues are shown as sticks and labeled. The residues mutated in only the first design strategy, summarized in Table I, are colored blue, and the residues that were also mutated in the second design strategy, summarized in Table 2, are colored purple. Figures made with PyMol http://www.pymol.org.

\section{Results \\ SEC binding assays can detect stabilized protein complexes in the micromolar-to-nanomolar range}

Steinle et al. observed that recombinantly produced and refolded NKG2D, mixed with wild-type MICA and injected onto an SEC column, eluted as an early shoulder to the unbound protein peak, at a molecular weight corresponding to a $\sim 60 \mathrm{kDa}$ complex rather than its individual 30 kDa components. [6] We observed the same results with our preparations of wild-type MICA mixed with 

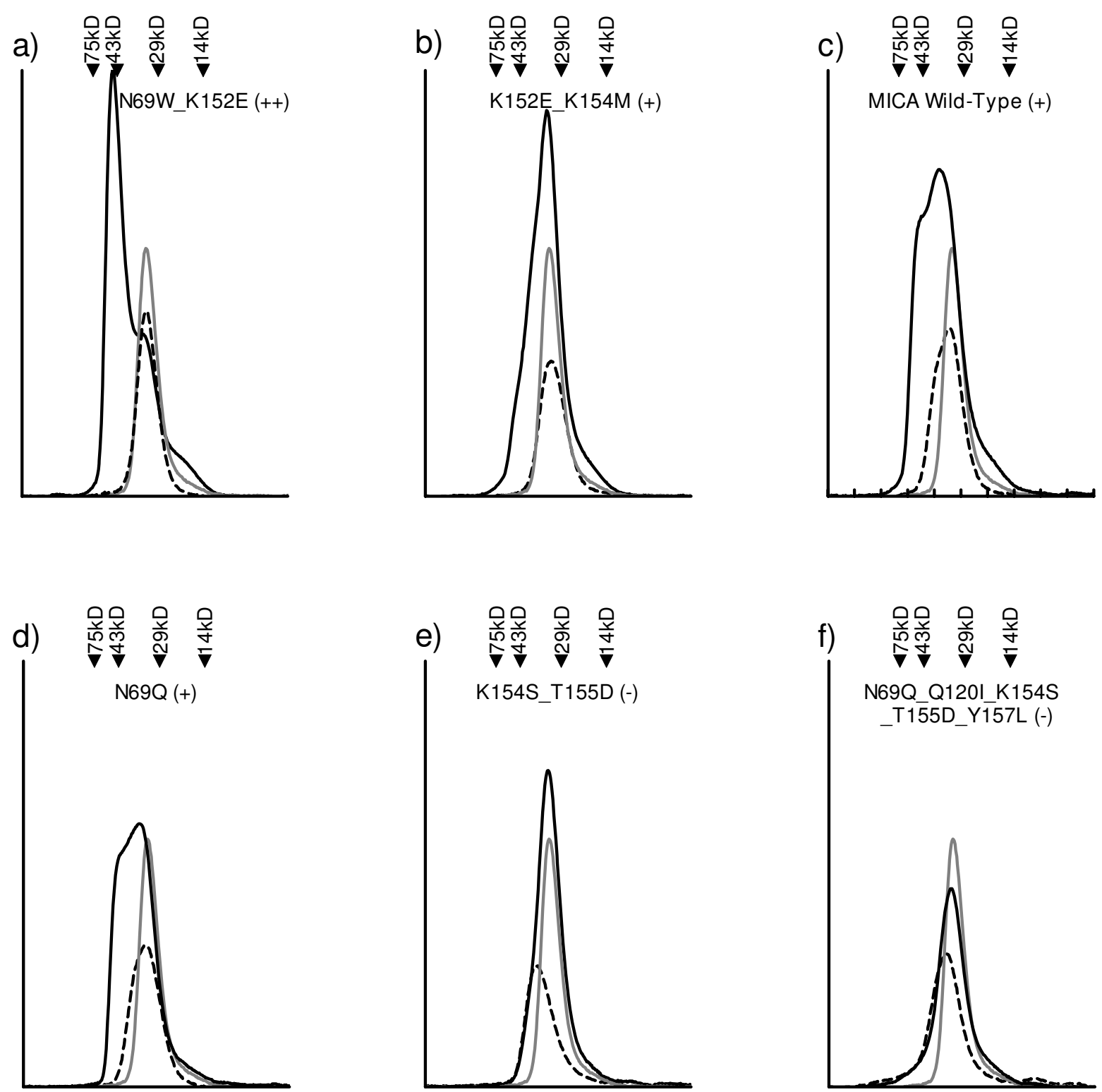

\section{Figure 2}

Differences among NKG2D-mutant MICA complexes as observed in the SEC binding assay. The elution profile of UV absorbance vs. time as constant flow resulting from an injection of NKG2D alone is shown as a gray line; MICA mutant or wild-type alone is shown as a dashed line; and NKG2D and MICA mutant or wild-type mixed in 2:I molar ratios is shown as a solid line. The elution times of size standards under identical conditions are shown above each graph. Graphs are labelled with the specific representative mutant. (a) Some mutants elute as a $\sim 60-k D a$ peak (++). (b-d) Some MICA mutants and wild-type elute with an early shoulder undifferentiated from the $\sim 30-k D a$ unbound peak $(+)$. (e-f) Some MICA mutants with stabilizing mutations elute only as a $\sim 30-\mathrm{kDa}$ peak aligned with the elution time of both proteins alone (-). 

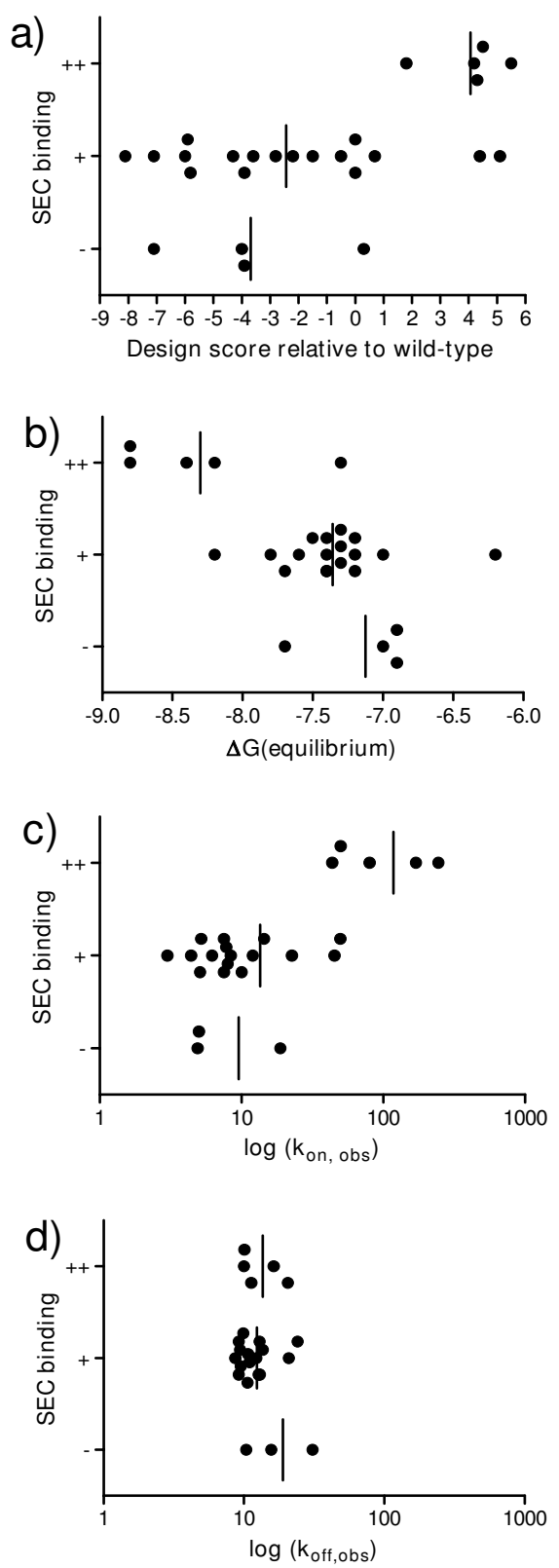

\section{Figure 3}

Comparison of SPR and SEC binding assays for evaluation of redesigned MICA proteins. Each MICA mutant from Table $\mathrm{I}$ is organized according to the results of the SEC binding assay and graphed against a different parameter. The average of each category is shown as a vertical line. (a) SEC results compared to RosettaDesign score [13] shows that negative RosettaDesign scores (predicted stabilized) only have binding results similar to or worse than wild-type, while positive RosettaDesign scores (predicted destabilized) include all 5 MICA mutants found to bind more tightly than wild-type. SEC results compared to SPR results [13] from (b) equilibrium and (c-d) kinetic analysis show similar correlations between equilibrium affinity and onrate with the highest-affinity mutants by SEC, while no correlation with off-rate is seen because the set of mutants does not vary significantly in this parameter. Observed $\mathrm{k}_{\text {on }}$ and $\mathrm{k}_{\text {off }}$ rates from single-step kinetic fits for mutants predicted to be stabilized, and from two-step kinetic fits for mutants predicted to be destabilized $\left(k_{+1}\right.$ and $k_{-2}$, respectively). ( $P$ values for the difference between ++ and + complexes: t-test $P=0.0013$ for Figure 3a; 0.0006 for Figure 3b; 0.000 I for Figure 3c; and 0.6 for Figure 3d.) 
homodimeric NKG2D (Figure 2c), and repeated these conditions for our set of redesigned MICA mutants (Figure 2). Some MICA mutants with high design scores (predicting MICA destabilization) mixed in a 1:2 molar ratio with NKG2D eluted as a separate, early $60 \mathrm{kDa}$ peak (Figure 2a). When this early peak was collected and analyzed by reducing SDS-PAGE, protein bands were observed at the molecular weights corresponding to both MICA (30 $\mathrm{kDa}$ ) and NKG2D (15 kDa). Other MICA mutants mixed with NKG2D eluted as early shoulders to the $30-\mathrm{kDa}$ peak (Figure $2 \mathrm{~b}$ and $2 \mathrm{~d}$ ), or as symmetrical $\sim 30$-kDa peaks (Figure $2 \mathrm{e}$ and $2 \mathrm{f}$ ). A qualitative correlation can be observed between SPR equilibrium $\Delta \mathrm{G}$ of binding and SEC bind assay results: Observation of an early shoulder corresponds to a low-micromolar range of affinity like wildtype MICA binding NKG2D. A differentiated early peak corresponds to high-nanomolar binding, as with MIC_N69W_K152E, and the absence of an early peak corresponds to low-to-mid-micromolar binding, as with MIC_N69Q_Q120I_K154S_T155D_Y157L.

Table I: SEC evaluation of predicted stabilized or mildly destabilized MICA mutants

\begin{tabular}{|c|c|c|}
\hline MIC Mutant & Design Score & SEC Binding \\
\hline N69Q_Q108L_Q120I_KI54S_TI55D & -8.1 & + \\
\hline N69Q_QI20I_KI54S_TI55D_YI57L & -7.1 & - \\
\hline N69Q_D72F_KI54S_TI55D & -7.1 & + \\
\hline N69Q_QI20I_KI54S_TI55D & -6 & + \\
\hline N69Q_D72F_Q108L_KI52V_YI57L & -5.9 & + \\
\hline KI52V_KI54S_TI55D_YI57L & -5.8 & + \\
\hline N69Q_KI54D & -4.3 & + \\
\hline KI54S_TI55D & -4 & - \\
\hline KI52V_KI54S_TI55D & -3.9 & - \\
\hline N69Q_D72F_Q_108L & -3.9 & + \\
\hline N69Q_D72F & -3.6 & + \\
\hline N69Q & -2.8 & + \\
\hline KI52V_YI57L & -2.2 & + \\
\hline KI54D & -1.5 & + \\
\hline Q108L & -0.5 & + \\
\hline Wild-type & 0 & + \\
\hline $\mathrm{D} 72 \mathrm{~W}$ & 0.3 & - \\
\hline Q120I & 0.7 & + \\
\hline Q120I_KI54M & 0.8 & $\mathrm{n} / \mathrm{a}$ \\
\hline N69W & 1.8 & ++ \\
\hline N69W_KI52E_KI54S & 4.2 & ++ \\
\hline N69W_KI52E_KI54D & 4.3 & ++ \\
\hline KI52E_KI54M & 4.4 & + \\
\hline N69W_D72F_KI52E & 4.5 & ++ \\
\hline N69W_D72W_KI52E & 5.1 & + \\
\hline N69W_KI52E & 5.5 & ++ \\
\hline
\end{tabular}

Design scores are listed relative to wild-type. Results of SEC binding assay follow the examples in Figure 2; n/a, not applicable because unbound mutant did not elute from column due to non-specific binding.

\section{SEC binding assays can detect differences in protein- protein on-rates}

The SPR study showed that some high design scores are associated with high affinity, and the SEC binding assay leads to the same conclusion (Figure 3). Equilibrium affinity agrees with the sets of protein binding strengths described by the SEC binding assay. Our design strategy altered non-contacting residues that did not change the off-rates, but did cause a large difference in on-rates. The SEC binding assay detected this, discriminating among complexes that had significantly varying on-rates, and the off-rates for this set of proteins were so similar that they were not a significant factor.

\section{Rationally designed MICA destabilization at single non- contacting residues does not enhance NKG2D binding}

A plot of NKG2D affinity vs. design score for the initial set of 25 MICA mutants produced previously shows that an area of design space was unfilled because the initial design strategy was biased toward stabilizing mutations and no MICA mutant was destabilized by more than 6 units. (Figure 4) After we found that mild MICA destabilization increased NKG2D affinity by more than an order of magnitude (Table 1), we then designed three more destabilizing mutants at the same locations to confirm if we were past the point of "diminishing returns," at which increased MICA destabilization would destabilize the complex with NKG2D. Using RosettaDesign, noncysteine residues were modeled at residues 108, 154, and 157. Point mutations predicted to provide a range of large disruptions to the wild-type structure were selected from the candidate designs produced by Rosetta, and prepared as previously reported [13]. In the SEC binding assay, none bound NKG2D better than wild-type MICA (Table 2). SPR equilibrium analysis showed their affinities were not significantly enhanced (Figure 4).

\section{Destabilized mutants elute early in analytical SEC}

In the SEC binding assay, the peak maximum of the unbound MICA mutant would decrease, moving from 3.5 $\mathrm{mL}$ for mutants with design scores of 50 or below to 3.1 $\mathrm{mL}$ for higher design scores (Figure 5), increasing apparent hydrodynamic radius from $23 \AA$ to $25 \AA$ (Table 2). When the column was saturated with buffer containing 1 $M$ urea, the two mutants with the largest predicted destabilizations continued to elute early (Figure 5). The earlyeluting shoulders for wild-type and the point-destabilized mutants were no longer observed. High-affinity MICN69W_K152E_K154D when mixed with NKG2D still eluted in $1 \mathrm{M}$ urea as an early peak (Table 2 ).

\section{Discussion}

In this study SEC formed the basis of a simple binding assay to assess protein-protein interaction strength, com- 
Table 2: SEC and SPR evaluation of predicted significantly destabilized MICA point mutants

\begin{tabular}{|c|c|c|c|c|c|c|}
\hline \multirow[b]{2}{*}{ MIC Mutant } & \multirow[b]{2}{*}{ Design Score } & \multirow[b]{2}{*}{$\underset{\left(\mathrm{kcal} \mathrm{mol}^{-1}\right)}{\Delta \mathrm{G}_{\mathrm{eq}}}$} & \multicolumn{4}{|c|}{ Buffer + 0 M Urea } \\
\hline & & & SEC Binding & $\begin{array}{c}\text { Elution } \\
\text { Volume }(\mathrm{mL})\end{array}$ & $\begin{array}{l}\text { Apparent } \\
M W(k D a)\end{array}$ & $\begin{array}{c}\text { Apparent } \\
r \\
(\AA)\end{array}$ \\
\hline WT & 0 & $-7.3 \pm 0.1$ & + & $3.5 \pm 0.1$ & $30 \pm 2$ & $23 \pm 2$ \\
\hline N69W_KI52E_KI54D & 4 & $-8.8 \pm 0.1$ & ++ & $3.4 \pm 0.1$ & $30 \pm 2$ & $23 \pm 2$ \\
\hline YI57W & 50 & $-7.9 \pm 0.1$ & - & $3.6 \pm 0.1$ & $30 \pm 2$ & $23 \pm 2$ \\
\hline KI54P & 220 & $-7.6 \pm 0.1$ & + & $3.1 \pm 0.1$ & $34 \pm 2$ & $25 \pm 2$ \\
\hline \multirow[t]{3}{*}{ Q108W } & 290 & $-7.8 \pm 0.2$ & + & $3.1 \pm 0.1$ & $34 \pm 2$ & $25 \pm 2$ \\
\hline & & & \multicolumn{4}{|c|}{ Buffer + I M Urea } \\
\hline & & & & Elution & Apparent & Apparent \\
\hline MIC Mutant & & & SEC Binding & Volume (mL) & $\begin{array}{l}M W \\
(\mathrm{kDa})\end{array}$ & $\begin{array}{c}r \\
(\AA)\end{array}$ \\
\hline WT & & & - & $2.8 \pm 0.1$ & $38 \pm 2$ & $27 \pm 3$ \\
\hline N69W_KI52E_KI54D & & & ++ & $2.9 \pm 0.1$ & $37 \pm 2$ & $26 \pm 3$ \\
\hline YI57W & & & - & $2.8 \pm 0.1$ & $39 \pm 2$ & $27 \pm 3$ \\
\hline KI54P & & & - & $2.6 \pm 0.1$ & $41 \pm 3$ & $28 \pm 3$ \\
\hline Q108W & & & - & $2.7 \pm 0.1$ & $40 \pm 3$ & $28 \pm 3$ \\
\hline
\end{tabular}

Design scores are listed relative to wild-type. $\Delta \mathrm{G}$ values determined at equilibrium by SPR. Wild-type and high-affinity MIC N69W_KI52E_KI54D SPR values as reported previously in [13]. Upper table shows SEC results with no added urea, and lower table shows SEC results in the presence of I M additional urea. Results of SEC binding assay follow the examples in Figure 2. Elution volume measured relative to the elution time of blue dextran. Apparent molecular weight (MW) and radii ( $r$ ) determined by line fit of elution time to Low Molecular Weight Standards (GE Healthcare). Standard errors are from triplicate experiments as described in Methods.

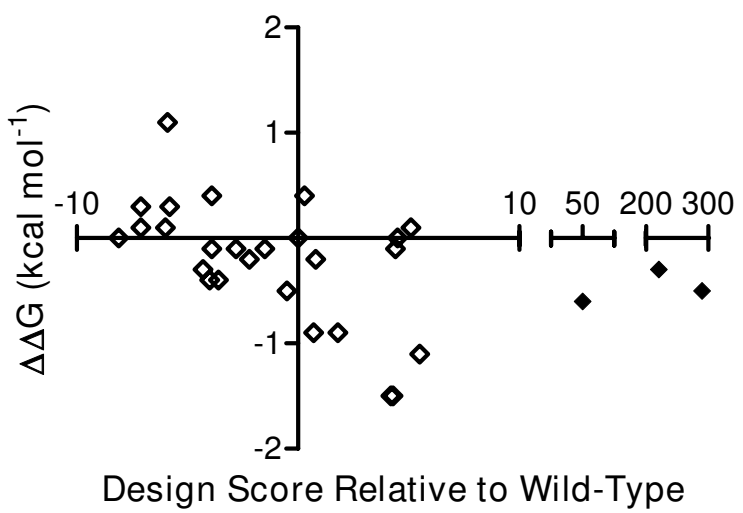

Figure 4

Comparison of the initial and follow-up design strategies. The difference in free energy of NKG2D binding relative to wild-type MICA is shown for each MICA mutant tested by SPR, from affinities determined at equilibrium. The RosettaDesign score relative to wild-type is plotted on the $x$ axis. MICA mutants in the initial design set (internal stabilization or destabilization; Table I) are shown as open diamonds. MICA mutants from the follow-up design set (significant internal destabilization; Table 2) are shown as filled diamonds. plementary to SPR binding assays. SEC results alone could evaluate the qualitative success or failure of exploring these regions of design space, although without quantitative kinetic or thermodynamic detail. The correlation of SEC and SPR is similar to the correlation between SEC and analytical ultracentrifugation (AUC) in that results from the two techniques generally agree, with SEC using common equipment, but AUC considered the "gold standard" [10,15-18]. AUC requires dedicated instrumentation, while SEC uses protein purification equipment and is faster [9]. SEC may be used to bring protein design to undergraduate research programs with limited equipment budgets, where the same pump used for protein chromatography during purification can be used for design evaluation through an SEC binding assay. The range of affinities that could be discriminated by the SEC binding assay was appropriate to design of the low-micromolar MICA-NKG2D interaction. Addition of $1 \mathrm{M}$ urea to the assay tuned it to be less sensitive to lower-affinity binding, while still discriminating between our most successful design and other candidates.

The relationship between elution time and protein-protein affinity has been modelled for small-zone SEC emphasizing the role of the off-rate in determining complex persistence. $[4,5]$ Our data show that SEC can dis- 


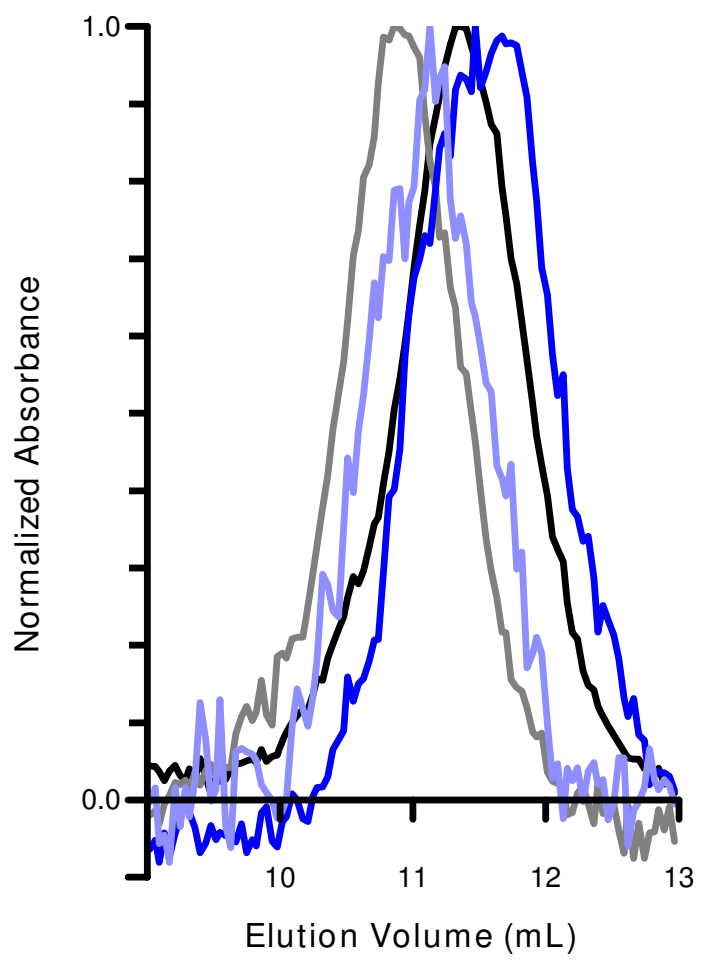

Figure 5

Effect of I M urea on the elution profiles of MICA wild-type and MIC_Q I 08W. Elution profiles of normalized UV absorbance vs. elution volume for injections of MICA wild-type (black) and MIC_QI08W (blue) in HBS-EA; and for MICA wild-type (gray) and MIC_Q I08W (light blue) in HBS-EA with I $M$ urea added.

criminate among complexes of different affinities that primarily differ in on-rate. We hypothesize that a fast onrate can allow a complex to re-attach before substantial separation, so that the complex remains in a small zone, even if the off-rate is fast.

Unbound mutant proteins with point mutations that are predicted to cause large destabilizations appear slightly larger than wild-type MICA by SEC. The average hydrodynamic radius of these proteins appears increased by destabilization; a similar observation using SEC-light scattering led to the conclusion that the protein in question was partially denatured [16]. For partially disordered proteins, the interplay between disorder and affinity is incompletely understood. Research into the relationship between dynamics and affinity may delineate which types of disorder promote binding, such as fly-casting $[19,20]$ or ground-state destabilization [21], and which types of disorder inhibit binding.

\section{Conclusion}

While the specific dynamic and structural impacts of the destabilizing mutations are only hinted at by the increased elution times of the SEC binding assay, the impact of these mutations on binding affinity is clear, in that none are stabilized in NKG2D affinity relative to wild-type MICA by more than $0.5 \mathrm{kcal} / \mathrm{mol}$, and none persist through the column as a bound complex. We decided from these results to target other regions for future design. The persistence of a high-affinity complex through the column even in the presence of $1 \mathrm{M}$ urea could imply significantly increased persistence time in the biological environment as well.

\section{Methods}

\section{Protein design and production}

The set of mutants in Table 1 was designed and produced as described previously [13]. Because the initial design strategy focused on increasing the stability of MICA in the region of the disordered loop, only 603 of 4608 designs were predicted to destabilize the receptor-bound conformation relative to wild-type. The set of mutants in Table 2 was designed to produce more destabilizing mutants. RosettaDesign [2] v2.0 was used with the coordinates of receptor-bound MICA from the NKG2D-MICA crystal structure (PDB ID 1HYR) [7]. Three of the eight previously altered locations at the center of the disordered region located on different structural elements were chosen (Figure $1 \mathrm{~b}$ ). RosettaDesign was used to model the results of mutating of each of these three residues to the 19 noncysteine amino acids with the backbone fixed to the receptor-bound coordinates. Mutations with large positive scores relative to wild-type were chosen for analysis.

Proteins were produced as described previously [13]. Before use in binding assays the proteins were dialyzed into HBS-EA buffer (10 mM HEPES [pH 7.4], $150 \mathrm{mM}$ $\mathrm{NaCl}, 3 \mathrm{mM}$ EDTA, and $0.02 \%$ sodium azide). Protein concentrations were determined by Nanodrop ND-1000 (Thermo Scientific) absorbance at $280 \mathrm{~nm}$ and by bicinchoninic (BCA) assay (Thermo Scientific).

\section{SEC binding assays}

$20 \mu$ mol each of homodimeric NKG2D and the MIC-A mutant were combined with HBS-EA buffer to a volume of $300 \mu \mathrm{L}$, mixed and immediately injected onto a Superdex 75 10/300 gel-filtration column (GE Healthcare) with a $500-\mu \mathrm{L}$ loop at $0.5 \mathrm{~mL} / \mathrm{min}$ using an AKTA PrimePlus FPLC system (GE Healthcare). The eluting protein was detected by UV absorbance at $280 \mathrm{~nm}$. These were compared to injections of $20 \mu \mathrm{mol}$ of homodimeric NKG2D or MICA alone. Columns were calibrated with Gel Filtration Calibration LMW standards (GE Healthcare: conalbumin, ovalbumin, carbonic anhydrase, and ribonuclease 
A, and blue dextran for void volume determination; also Sigma: cytochrome c). Several individual proteins and mixed receptor-ligand complexes were injected three or more times onto the same column, resulting in variations in elution volume of no more than $0.1 \mathrm{~mL}$. Fractions were collected using the AKTA Prime fraction collector for analysis by reducing analytical SDS-PAGE using Coomassie Blue staining. Observed variations in elution volume for triplicate experiments with urea in the buffer were as listed in Table 2.

\section{SPR binding assays}

For the first set of mutants (Table 1), determination of NKG2D-mutant MICA kinetics and thermodynamics by SPR was previously described [13]. NKG2D affinities for the second set of MICA mutants (Table 2) were determined by equilibrium binding analysis as used in the previous study. (Fast kinetics precluded the use of kinetic fits.) Standard errors reported in the data tables result from triplicate (or more) experiments.

\section{Competing interests}

The authors declare that they have no competing interests.

\section{Authors' contributions}

CLM and WKS carried out SEC binding assays. WKS and BJM carried out SPR binding assays. CLM, WKS, MAS, ADV, and CRA designed, made and purified mutant proteins. All authors participated in interpretation of the data. CLM, WKS, CRA and BJM drafted the manuscript. All authors have read and approved the final manuscript.

\section{Acknowledgements}

We thank Derek Wood and Kathryn Houmiel (Seattle Pacific University) for technical assistance and early evaluation of this research as student presentations, Tanja Kortemme (UCSF) for the initial design with Rosetta, and Roland Strong (Fred Hutchinson Cancer Research Center) for instrument use. This research was funded by National Institutes of Health Grant RI5 Al058972.

\section{References}

I. Kortemme T, Baker D: Computational design of protein-protein interactions. Curr Opin Chem Biol 2004, 8(I):9l-7.

2. Kortemme T, Baker D: A simple physical model for binding energy hot spots in protein-protein complexes. Proceedings of the National Academy of Sciences of the United States of America 2002, 99(22): |4||6-|4|2|.

3. Winzor DJ: Quantitative characterization of ligand binding by chromatography. In Protein-Ligand Interactions: Hydrodynamics and Calorimetry: A Practical Approach Edited by: Harding SE, Chowdhry BZ. Oxford: Oxford University Press; 2001:47-74.

4. Stevens FJ: Analysis of protein-protein interaction by simulation of small-zone size exclusion chromatography. Stochastic formulation of kinetic rate contributions to observed high-performance liquid chromatography elution characteristics. Biophys J 1989, 55(6): I I55-I I67.

5. Wilton R, Myatt EA, Stevens FJ: Analysis of protein-protein interactions by simulation of small-zone gel filtration chromatography. In Protein-Protein Interactions: Methods and Protocols Volume 26I. Edited by: Fu H. Totowa, NJ: Humana Press Inc; 2004: I37-I54.

6. Steinle A, Li P, Morris DL, Groh V, Lanier LL, Strong RK, Spies T: Interactions of human NKG2D with its ligands MICA, MICB, and homologs of the mouse RAE-I protein family. Immunogenetics 200I, 53(4):279-287.

7. Li P, Morris DL, Willcox BE, Steinle A, Spies T, Strong RK: Complex structure of the activating immunoreceptor NKG2D and its MHC class I-like ligand MICA. Nat Immunol 200I, 2(5):443-45 I.

8. Franzini M, Bramanti E, Ottaviano V, Ghiri E, Scatena F, Barsacchi R, Pompella A, Donato L, Emdin M, Paolicchi A: A high performance gel filtration chromatography method for gamma-glutamyltransferase fraction analysis. Anal Biochem 2008, 374(I): I-6.

9. le Maire M, Arnou B, Olesen C, Georgin D, Ebel C, Moller JV: Gel chromatography and analytical ultracentrifugation to determine the extent of detergent binding and aggregation, and Stokes radius of membrane proteins using sarcoplasmic reticulum Ca2+-ATPase as an example. Nat Protoc 2008, 3(I I): I782-95.

10. Gralle M, Oliveira CL, Guerreiro LH, McKinstry WJ, Galatis D, Masters CL, Cappai R, Parker MW, Ramos CH, Torriani I, et al.: Solution conformation and heparin-induced dimerization of the fulllength extracellular domain of the human amyloid precursor protein. J Mol Biol 2006, 357(2):493-508.

II. Copeland RA, Pompliano DL, Meek TD: Drug-target residence time and its implications for lead optimization. Nat Rev Drug Discov 2006, 5(9):730-9.

12. Tummino PJ, Copeland RA: Residence time of receptor-ligand complexes and its effect on biological function. Biochemistry 2008, 47(20):548I-5492.

13. Lengyel CS, Willis LJ, Mann P, Baker D, Kortemme T, Strong RK, McFarland BJ: Mutations designed to destabilize the receptorbound conformation increase MICA-NKG2D association rate and affinity. J Biol Chem 2007, 282(42):30658-30666.

14. Strong RK, McFarland BJ: NKG2D and Related Immunoreceptors. Adv Protein Chem 2004, 68:28I-3I 2.

15. Berkowitz SA: Role of analytical ultracentrifugation in assessing the aggregation of protein biopharmaceuticals. AAPS J 2006, 8(3): E590-605.

16. Philo JS: Is any measurement method optimal for all aggregate sizes and types? AAPS J 2006, 8(3):E564-7I.

17. Gualfetti PJ, Iwakura M, Lee JC, Kihara H, Bilsel O, Zitzewitz JA, Matthews CR: Apparent radii of the native, stable intermediates and unfolded conformers of the alpha-subunit of tryptophan synthase from E. coli, a TIM barrel protein. Biochemistry 1999, 38(40): $13367-13378$

18. Gabrielson JP, Brader ML, Pekar AH, Mathis KB, Winter G, Carpenter $\mathrm{JF}$, Randolph TW: Quantitation of aggregate levels in a recombinant humanized monoclonal antibody formulation by sizeexclusion chromatography, asymmetrical flow field flow fractionation, and sedimentation velocity. J Pharm Sci 2007, 96(2):268-79.

19. Hoffman RM, Blumenschein TM, Sykes BD: An interplay between protein disorder and structure confers the $\mathrm{Ca} 2+$ regulation of striated muscle. J Mol Biol 2006, 36 I (4):625-33.

20. Shoemaker BA, Portman JJ, Wolynes PG: Speeding molecular recognition by using the folding funnel: the fly-casting mechanism. Proceedings of the National Academy of Sciences of the United States of America 2000, 97(16):8868-8873.

21. Horn JR, Kraybill B, Petro EJ, Coales SJ, Morrow JA, Hamuro Y, Kossiakoff $A A$ : The role of protein dynamics in increasing binding affinity for an engineered protein-protein interaction established by H/D exchange mass spectrometry. Biochemistry 2006, 45(28):8488-8498. 